\title{
AI-Supply chain Risk Management during Pandemic
}

\author{
Dhanesh Thatikonda
}

\begin{abstract}
Artificial intelligence (AI) was introduced to develop and create "thinking machines" that are capable of mimicking, learning, and replacing human intelligence. Since the late 1970s, AI has shown great promise in improving human decision-making processes and the subsequent productivity in various business endeavors due to its ability to recognize business patterns, learn business phenomena, seek information, and analyze data intelligently. Despite its widespread acceptance as a decision-aid tool, AI has seen limited application in supply chain management (SCM). To fully exploit the potential benefits of AI for SCM, this paper explores various sub-fields of AI that are most suitable for solving practical problems relevant to SCM. In so doing, this paper reviews the past record of success in AI applications to SCM and identifies the most fruitful areas of SCM in which to apply AI.
\end{abstract}

Index Terms - Artificial Intelligence, Supply Chain Management, Knowledge Management, Risk Management.

\section{INTRODUCTION}

Enterprise applications based on advanced technologies such as AI and machine learning (ML), though still in the nascent stages of development, are beginning to drive innovation strategies of business. In the supply-chain and logistics industry, these technologies are proving to be a game changer. McKinsey \& Company expects businesses to gain between $\$ 1.3$ tr and $\$ 2$ tr a year in economic value by using AI in their Supply Chains.

According to PricewaterhouseCoopers, AI could contribute almost $\$ 15.7$ tr to the global economy by 2030 .

\section{AI SUPPLY CHAIN MANAGEMENT}

AI adoption is taking off in the supply chain is the realization by companies of its potential to solve the complexities of running a global logistics network. Implemented correctly, AI helps companies to make smarter and more agile decisions and anticipate problems. Proactive systems enabled by AI are raising the quality of service, exceeding customer expectations for on-time and undamaged deliveries. They're further improving efficiency through automated compliance processing. The result is lower costs and fewer problems across the logistics network. Fig. 1 shows AI presence in entire supply chain.

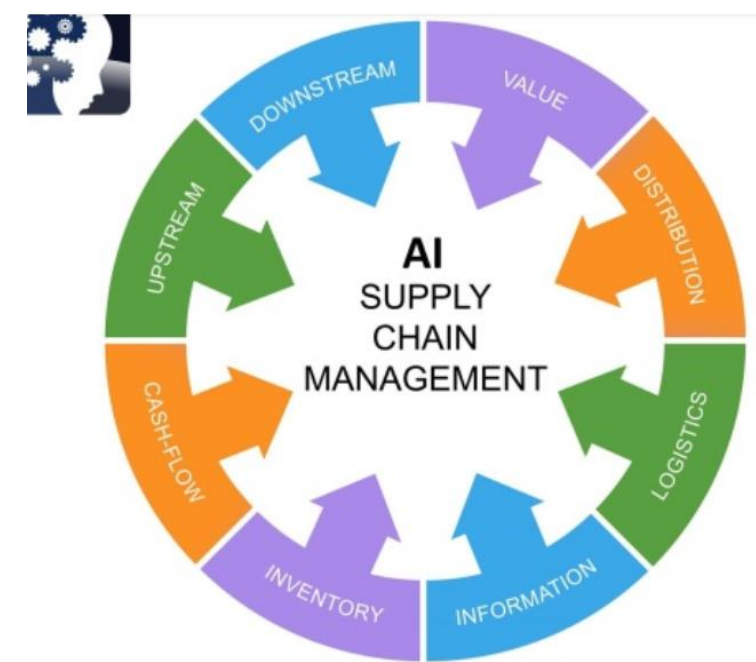

Fig. 1. AI Integrated Supply Chain Management.

\section{PREDiCTIVE CAPABILITIES ARE HELPING DEMAND FORECASTING}

When inventory lags demand, companies suffer losses. AI is ramping up efficiencies in network planning and predictive demand, allowing merchandisers to become more proactive. By knowing what to expect, they can adjust the number of vehicles and direct them to locations where maximum demand is expected. This leads to lower operational costs.

\section{IV.CHATBOTS ARE REDEFINING CUSTOMER SUPPORT}

AI can personalize the relationship between customers and logistics providers. A recent example of personalized customer experience is DHL's partnership with Amazon. By activating DHL Parcel "skill" via the Alexa app, DHL customers can ask Alexa to connect with Amazon Echo or Echo Dot smart speakers and confirm their parcels' status. In the event of any issues arising during the interaction, Echo users can directly contact DHL for assistance by its customersupport team.

\section{SMart Warehouses Are More EFFICIENT}

A smart warehouse is a fully automated facility wherein most work is done through automation or software. In the process, tedious tasks are simplified, and operations become more cost-effective. Alibaba and Amazon have already transformed their warehouses using automation. Amazon recently rolled out machines that automate the job of boxing customer orders. In Amazon warehouses, robots work alongside humans to increase productivity and efficiency. 


\section{GENERIC ALGORITHMS ARE IMPROVING DELIVERY Times AND REDUCING COSTS}

In the logistics business, every mile and minute matters. Companies can use a route planner based on genetic algorithms to map out optimal routes for deliveries.

\section{SUPPLY CHAIN RISK MANAGEMENT}

SCRM as an academic topic was identified as important in 2001-2002 and has since gained extensive recognition as an important topic both within academia and industry. With changing weather patterns, natural disasters, political turmoil, financial upheavals, and now a pandemic, supply chains are under constant pressure to deliver. The basic challenge in the scenarios mentioned above is the aspect of supply disruption. In general, the focus in most cases is to strengthen the upstream supply chain to maintain continuity of supply. However, as seen in this pandemic the cause of the risk upstream was the lack of human resource due to the lockdown (with operations shut) and an unknown demand profile due to stockpiling on one end coupled with reduced footfall in the retail environment on the other. SCRM in general borrows from risk management techniques from multidisciplinary sources such as engineering, finance, and mathematics. There is an increasing amount of literature on SCRM, but the basic process is comprised of three activities: identification of the risk, quantification of the risk, and identifying potential solutions to mitigate the impact of the risk. Simplistically, risk can be defined as an event that has the probability of creating a negative impact for the organization or supply chain. There are a number of papers and articles that focus on the sources of risk (for example, whether the risks are external or internal to the firm, whether the risks are upstream or downstream) the different quantification techniques (for example, risk trees, FMEA), and the solutions for mitigating the impact of the risks (transferring the risk, hedging, postponing the risk, avoiding the risk).

Another aspect of SCRM is to consider both proactive management - creating systems in anticipation of the risk to mitigate; as well as reactive management - reacting quickly to minimize the impact of the risk. Fig. 2 describes the reactive risk management steps.

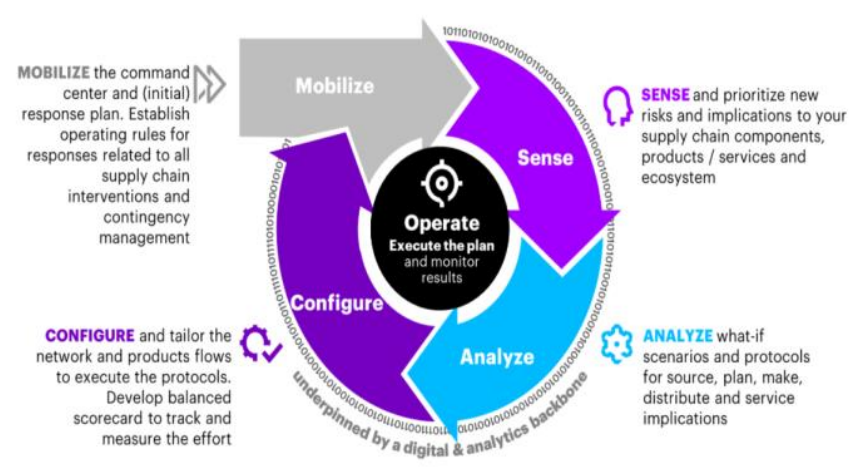

Fig. 2. Reactive Risk Management Response.

\section{COVID-19}

Pandemic has driven home the need to reduce global supply chain vulnerabilities through intelligent workflows. As the world works to manage the current COVID-19 outbreak (see Fig. 3), the focus is rightly on communities treating those infected, companies protecting employees worldwide, and collectively containing the virus to limit global health impact.

It's clear that economic and societal costs will be huge. The pandemic has revealed the vulnerabilities and fragilities in global supply chains across most, if not all, sectors, and industries. Today, organizations are in reaction mode, focused strictly on maintaining supply and meeting customer needs, often through hands-on rigor and hard work.

However, at some point, leaders must analyze the current pain points to better prepare for tomorrow. To avoid perpetual reaction to future "black swan" situations, leaders need to evaluate how they can proactively get ready for future unpredictable, yet inevitable, disruptions.

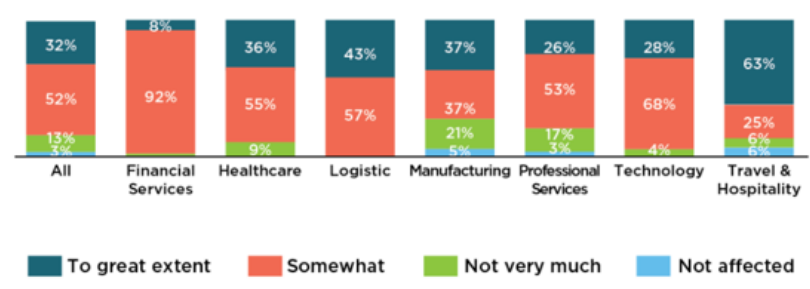

Fig. 3. Impact of covid-19 to business operations in Asia Pacific.

\section{AI AND SCRM}

Supply chains are complex systems comprising of various organizations, operations, processes, assets, people, and strategic stakeholders. The complexity is also manifested through the information flowing across the chain which ensures that appropriate decisions are taken for the supply chain to work both efficiently and effectively. These decisions are taken by humans in the chain. As supply chains have gone far and wide there is even more complexity due to unpredictable weather patterns and external sources of risks.

However, software systems have grown in capability and with big data systems there is a lot of data available for both pattern analysis and decision making. To add to the big data available from supply chain software systems, there is a plethora of dynamic fast data from social media streams that provides valuable information about ground reality of locations, assets, sentiments, and weather which has the potential to be tapped for better decision making.

The use of AI and specifically machine learning techniques could be used to harness both big data and fast data from the supply chain systems to predict supply chain risks and prescribe mitigating strategies. The future may be a situation where the system takes over the mitigation process after the decision is made, without human intervention. The research is moving in that direction, but from a SCRM perspective the ability of the humans to control and mitigate the risks will be more important. 
AI systems will be able to identify the source of risk (ideally predict it from big data analytics), quantify the risk based on past impact data, and suggest the best possible mitigation strategies for that scenario (See Fig. 4) . As discussed above, this method is a regular process, however $\mathrm{AI}$, and data analytics will help provide a faster analysis process, a faster quantification process, and perhaps better suggestions for mitigation based on a wide ranging analysis of past scenarios. For example, if we consider a scenario of sourcing grain from farms, the system could analyze weather patterns, predict which farms could be affected in the future (or near future) and do a portfolio analysis to redesign upstream sourcing to minimize supply chain disruption by identifying the appropriate suppliers from the portfolio. This could also work based on analyzing supplier quality data. In another scenario, if a supplier location is affected by a local or regional upheaval, the system could analyze big data and fast data (from social media feeds) to decide the severity of the impact, and then decide whether a supply chain redesign is required for business continuity.

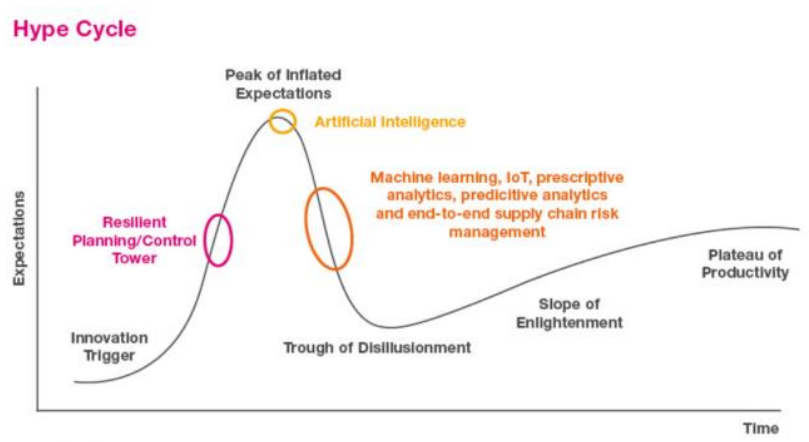

Fig. 4. AI and Machine learning influencing peak expectations.

In the future, sustainability non-conformance will be a major supply chain risk and AI systems will need to be taught about sustainability parameters and its importance within supply chains.

It is clear from the discussion that the capability of the system will depend upon the AI algorithms and the available data. Data availability and appropriate data is critical for the system to manage supply chain risks effectively. There is debate within the community about whether the AI systems should be like humans or should be designed to naturally remove all bias as ingrained in humans. Since the discussion will continue until the capability is fully formed, it is important that the AI (Machine Learning) system provides the following characteristics at the present:

Explainability - the ability to explain how the AI system is working.

Interpretability - the extent to which one can predict what will happen if there is a change in the input to the system or parameters in the algorithm, the ability to interpret how the system is working.

Auditability - the ability to audit the system, the data and how this data is used.

Transparency - the characteristics that provides full access to how the system works and provides trust to all stakeholders.

\section{CONCLUSION}

Companies like Amazon, Walmart, Alibaba, and Zara are leading the path in the use of technology and advanced analytics in managing supply chains. Artificial Intelligence $(\mathrm{AI})$ and Machine Learning (ML) are of the hot topics being discussed in the retail world. Across the globe, whether they are grocery, mass merchant, or fashion, retailers are looking for ways to make faster and smarter decisions using this technology. Among the leading applications of AI \& ML in planning are forecasting and replenishment processes.

\section{REFERENCES}

[1] Thatikonda, Dhanesh. "Recent Information Technology Trend of Using Machine Learning in Manufacturing Scheduling." International Journal of Science and Research (IJSR) 9, no. 7 (2020): 1087-88. doi:10.21275/SR20712050357.

[2] Meng, Fanwen. "Supply Chain Risk Management." International Journal of Information and Decision Sciences (2008): n. pag. Print.

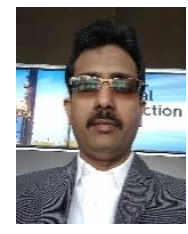

Dhanesh Thatikonda is a Senior Product Manager at TMobile USA, having 16+ years of design, development experience in Supply Chain Management Applications. $\mathrm{He}$ has vast domain experience in Retail and Manufacturing Organizations. He has conducted several trainings and published several articles in International Journals. 